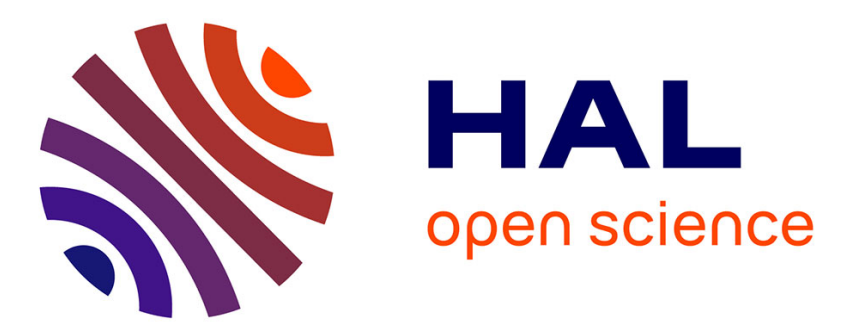

\title{
Heat transport in semiconductor crystals: Beyond the local-linear approximation
}

\author{
Younès Ezzahri, Karl Joulain, Jose Ordonez-Miranda
}

\section{To cite this version:}

Younès Ezzahri, Karl Joulain, Jose Ordonez-Miranda. Heat transport in semiconductor crystals: Beyond the local-linear approximation. Journal of Applied Physics, 2020, 128, 10.1063/5.0017188 . hal-03017603

\section{HAL Id: hal-03017603 https://hal.science/hal-03017603}

Submitted on 21 Nov 2020

HAL is a multi-disciplinary open access archive for the deposit and dissemination of scientific research documents, whether they are published or not. The documents may come from teaching and research institutions in France or abroad, or from public or private research centers.
L'archive ouverte pluridisciplinaire HAL, est destinée au dépôt et à la diffusion de documents scientifiques de niveau recherche, publiés ou non, émanant des établissements d'enseignement et de recherche français ou étrangers, des laboratoires publics ou privés. 


\section{Heat transport in semiconductor crystals: Beyond the local-linear approximation}

Cite as: J. Appl. Phys. 128, 105104 (2020); https://doi.org/10.1063/5.0017188

Submitted: 08 June 2020 . Accepted: 19 August 2020. Published Online: 08 September 2020

Younès Ezzahri (D), Karl Joulain (D), and José Ordonez-Miranda (D)

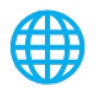

\section{Lock-in Amplifiers up to $600 \mathrm{MHz}$}
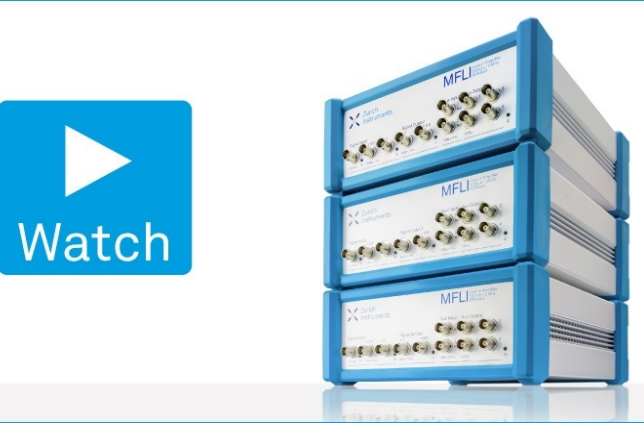

J. Appl. Phys. 128, 105104 (2020); https://doi.org/10.1063/5.0017188 128, 105104 


\title{
Heat transport in semiconductor crystals: Beyond the local-linear approximation
}

\author{
Cite as: J. Appl. Phys. 128, 105104 (2020); doi: 10.1063/5.0017188 \\ Submitted: 8 June 2020 . Accepted: 19 August 2020 . \\ Published Online: 8 September 2020
}

Younès Ezzahri, ${ }^{\text {) }}$ (D) Karl Joulain, (D) and José Ordonez-Miranda (D)

\begin{abstract}
AFFILIATIONS
Institut Pprime, CNRS, Université de Poitiers, ISAE-ENSMA, F-86962 Futuroscope Chassneuil, France
\end{abstract}

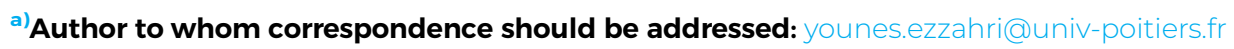

\begin{abstract}
We extend the application of the nonlocal theory of Mahan and Claro [Phys. Rev. B 38, 1963 (1988)] to solve the steady-state BoltzmannPeierls transport equation within the framework of the single mode relaxation time approximation using the modified Debye-Callaway model. We consider the case of a semi-infinite semiconductor (SC) crystal with a boundary condition at its top surface that can be considered reasonably representative of time domain thermoreflectance (TDTR) and frequency domain thermoreflectance (FDTR) techniques. The approach allows us to obtain three different contributions to the heat flux density current that shed further light on the fundamental role of nonlocality and nonlinearity in heat transport by phonons in SC crystals. Through their intrinsic and implicit shuffling effect of the crystal momentum, phonon-phonon Normal scattering processes play a key role in the onset of thermal conduction as they introduce the temperature Laplacian as a second driving potential force for the heat flux density current in addition to the conventional Fourier's temperature gradient. The developed model suits quite fairly to interpret the frequency behavior of the reduced effective thermal conductivity of SC crystals that is observed in TDTR and FDTR experiments. We obtain an expression of the effective thermal conductivity of the SC crystal that is characterized with a universal spectral suppression function that captures and describes the role, the weight, and the contribution of quasi-ballistic and non-diffusive phonons. The spectral suppression function only depends on the ratio between the phonon mean free path and the thermal penetration depth as defined based on the diffusive Fourier's law.
\end{abstract}

Published under license by AIP Publishing. https://doi.org/10.1063/5.0017188

\section{INTRODUCTION}

Due to the continuous development in nanotechnology and the rapid evolution in the synthesis and fabrication of different solid materials at a nanometer scale, understanding and controlling heat transport at very short length scales has become very crucial and challenging. Indeed, the length scales at which heat transport starts taking place in all types of conductor materials, e.g., metals, semiconductors (SCs) and dielectrics, undertake a continuous reduction to almost approach the intrinsic characteristic microscopic lengths of the material. ${ }^{1,2}$ For the purpose of heat transport in SC and dielectric materials, the main characteristic intrinsic length is the mean free path (MFP) of phonons that are the main energy (heat) carriers in these materials. ${ }^{3,4}$ In a local theory, heat transport depends only on the material properties and temperature gradient at a single point in space and is not affected by the properties and temperature profiles at other locations. The local theory of heat transport is valid as long as the MFP is short compared to the spatial variations in temperature. Nevertheless, because of the phonon dispersion relation, its MFP is inversely proportional to a certain power of its frequency $\omega,{ }^{5,6}$ and thus, no matter what distance scale one has for the temperature variation, there are always phonons of small $\omega$ with a longer MFP. Hence, the nonlocal theory would actually be needed in the analysis of all aspects of heat transport by phonons in SC and dielectric crystals, particularly when low frequency phonons have a significant contribution to the process.

The question of energy and heat transport mechanisms in solid materials at short time and length scales has been the basis of numerous theoretical and experimental papers. A part of the research has continued to exploit the conventional local/linear nonequilibrium thermodynamics theory (LLNETT), ${ }^{7-47}$ while the second part has considered going beyond this theory and has investigated the nonlocal/nonlinear effects using different approaches, especially in the presence of large temperature gradients. ${ }^{48-71}$ The difference between the two approaches resides in the relation between the temperature disturbance and the heat flux density 
current. In the LLNETT, the heat flux density current is a local and linear function of the temperature gradient $\nabla T$ that constitutes the only driving potential force. On the other hand, when nonlocal and nonlinear effects are considered, other driving potential forces come to play with two kinds of terms $\nabla^{n} T$ and $(\nabla T)^{n}$ with $n>1$; the former are nonlocal while the latter are nonlinear. ${ }^{48}$ Therefore, the difference lies in the nature of the driving potential forces and not in the inherent temperature dependence of their coefficients. This latter dependence leads to nonlinearity effects even in the conventional Fourier's law, which is a local/linear theory in the sense described above.

Among the different studies, the one of non-diffusive and quasiballistic phonon transport in SC and dielectric crystals including the transition between the ballistic and diffusive regimes has attracted a growing interest in the last decade. ${ }^{10,12,14-17,20-47,57-63,65,68-71}$ In fact, some recent works have shown that the average MFP of phonons in SC crystals can be up to two orders of magnitude longer than the prediction of the kinetic theory at room temperature. ${ }^{5,6}$ This has raised the fundamental question about the real contribution of low frequency phonons to the effective thermal conductivity of SC crystals that has been measured by several photothermal methods, mainly time domain thermoreflectance (TDTR), ${ }^{10,16,21,29,30,59,60,62,70}$ frequency domain thermoreflectance (FDTR), ${ }^{22,25}$ and transient thermal grating (TTG) $)^{17,20,31,35,41,45}$ techniques. The exploitation of these techniques has allowed obtaining the key and relevant information about the phonon MFP spectral distribution in SC crystals, especially at room temperature. ${ }^{16,17,20-22,25,29-31,35,41,45}$ The thermal conductivity accumulation function and the suppression function are two elegant metrics that have been used in the analysis of the experimental data in these configurations. The former is used for understanding which phonon MFPs contribute predominantly to heat transport in a material, ${ }^{32}$ while the latter is used to quantify the reduction or suppression of the thermal conductivity. ${ }^{14,27,39}$ The two metrics are actually related. The suppression function provides the ability to extend the notion of thermal conductivity beyond the diffusive regime in which it is defined from Fourier's law. ${ }^{14,27,39}$ By utilizing the suppression function for a given experimental geometry, one can obtain through a reconstruction method, such as the one proposed by Minnich, ${ }^{17}$ the material's phonon MFP spectral distribution from the experimentally measured thermal conductivity. ${ }^{17,22,25,29-31}$ To obtain the effective thermal conductivity, the thermal signal from the experiment is fitted to the results of Fourier's law. The suppression function is calculated through modeling of the given experimental geometry with the Boltzmann-Peierls transport equation (BPTE). As pointed out by Chiloyan et al., a key assumption in this method is the universality of the suppression function; the ability to express this function as a function of a single parameter, i.e., the ratio of the phonon MFP to a characteristic length for the given experimental configuration and not otherwise of the material properties.

The motivation behind the present work is twofold. First, we extend the application of the nonlocal theory of Mahan and Claro $^{48}$ to solve steady-state BPTE using the Callaway approximation of the collision operator. This will allow us calculating and developing a compact formula of the heat flux density current that shed further light on the fundamental role of nonlocality and nonlinearity in heat transport by phonons in SC crystals and therefore go beyond the conventional Fourier's law. A particular attention will be addressed toward the critical role of phonon-phonon Normal scattering processes. Second, we derive an analytical expression of the spectral suppression function of the effective thermal conductivity in TDTR and FDTR configurations and emphasize its universal character and features.

We present the main steps of the theoretical modeling in Sec. II. In Sec. III, we discuss the results of this approach in application to TDTR and FDTR results by analyzing the effect of varying different experimental parameters. We summarize and establish our concluding remarks in Sec. IV.

\section{THEORY}

In this section, we present the method that allows deriving the full expression of the phonon heat flux density current in cubic SC crystals that gives a clear insight into the fundamental role of nonlocality and nonlinearity effects. We assume the temperature profile to vary in only one dimension and consider a semi-infinite geometry for the SC crystal that corresponds to TDTR and FDTR experimental configurations. This permits obtaining a flexible and rather smooth analysis, as we shall see below.

\section{A. Boltzmann-Peierls transport equation}

The starting point of our modeling is steady-state BoltzmannPeierls transport equation (BPTE) in the framework of the single mode relaxation time approximation and using the modified Debye-Callaway model in which both longitudinal and transverse phonon modes are included explicitly. ${ }^{72-79}$ The SC crystal is assumed to have a cubic symmetry and is treated as a continuum, elastic, and isotropic medium characterized by a linear (Debye-like) phonon spectrum for each phonon branch polarization so that one considers heat transport due only to acoustic phonons and ignore any contribution from optical phonons. ${ }^{72-79}$ Callaway's approximation of the collision operator in BPTE captures quite fairly and respectfully the peculiar effects of phonon-phonon Normal scattering processes ( $\mathrm{N}$-processes) that distinguish them from the rest of phonon scattering processes including phonon-phonon Umklapp scattering processes (U-processes). Thus, it allows a simple separation of N-processes and U-processes. ${ }^{72}$ The pioneer purely intuitive work of Callaway ${ }^{72}$ was investigated in detail by many authors, and more robust theoretical foundations have been found. ${ }^{4,75,76}$ For their algebraically convenient forms, the Callaway ${ }^{72}$ and Holland ${ }^{74}$ methods have been the most and widely used formulations for the steady-state local/linear (Fourier's law based) thermal conductivity that enable fitting of the experimental data for a large number of materials in which heat is carried by phonons, with only a few adjustable parameters.

Despite its simplicity, the Debye-Callaway model has been proven to be very robust and effective in the study and prediction of the temperature behavior of the thermal conductivity of SC crystals within the conventional local/linear non-equilibrium thermodynamics theory. ${ }^{72-79}$ Although this model might not be very rigorous, the treatment is to some extent justified by the reasonable agreement with the experiment that has been obtained with it in the steady-state regime. ${ }^{77,78}$ In this approach, the contributions of longitudinal and transverse acoustic branch polarizations are considered separately; furthermore, any conversion of normal modes 
between both acoustic polarizations (inter-transitions) is neglected; only transitions within the same acoustic branch polarization (intra-transitions) are considered. This approach was first used by Holland $^{74}$ in his extension of the Callaway's model. ${ }^{72}$

We assume application of a temperature disturbance along the direction $\vec{x}$. In addition, we consider local thermal equilibrium throughout, which is required to define a temperature. ${ }^{3,4}$ Under the single mode relaxation time approximation, the steady-state Callaway's form of the BPTE along the $x$ axis can be written as follows:

$$
v_{p} m \frac{d U_{q, p}^{m}}{d x}=-\frac{U_{q, p}^{m}-U_{q, p}^{0}}{\tau_{q, p}^{C}}+\frac{g_{q, p}^{m}}{\tau_{q, p}^{C}},
$$

where we have introduced the deviational spectral energy density per phonon mode (phonon wave-packet) of wave-vector $q$ and polarization $p$ as $U(x, m, q, p) \equiv U_{q, p}^{m}=\hbar \omega_{p}\left(n_{q, p}^{m}-n_{q, p}^{E q}\right) . n_{q, p}^{m}$ is the phonon distribution function at the absolute local thermal equilibrium temperature $T$, and $U_{q, p}^{0}=\hbar \omega_{p}\left(n_{q, p}^{0}-n_{q, p}^{E q}\right)$ is therefore the deviational equilibrium spectral energy density per phonon mode with $n_{q, p}^{0}$ and $n_{q, p}^{E q}$ denoting the equilibrium phonon Planck distribution functions, at temperatures $T$ and $T_{0}$, respectively, where $T_{0}$ represents an absolute reference temperature. ${ }^{27,39}$

$$
\left(\tau_{q, p}^{C}\right)^{-1}=\left(\tau_{q, p}^{R}\right)^{-1}+\left(\tau_{q, p}^{N}\right)^{-1} \text { is the "combined" relaxation }
$$

time, ${ }^{72,73}$ with $\tau_{q, p}^{R}$ representing the single relaxation time with which all resistive phonon scattering processes (all scattering processes that change the total phonon wave-vector: Umklapp, boundary, defects, imperfections) tend to return the phonon system to its thermal equilibrium state. $\tau_{q, p}^{N}$ is the single relaxation time due to $\mathrm{N}$-processes (scattering processes that do not change the total phonon wave-vector). As pointed out by Callaway ${ }^{72}$ and others, ${ }^{73-76} \mathrm{~N}$-processes tend to return the phonon system to a displaced (drifted) Planck distribution function $n_{q, p}^{\lambda_{p}}=\left\{\exp \left[\frac{\left(\hbar \omega_{p}(q)-\lambda_{p} \cdot q\right)}{k_{B} T}\right]-1\right\}^{-1}$ where, by symmetry consideration in cubic SC crystals, $\lambda_{p}$ is a constant vector in the direction of the applied temperature disturbance which has the dimension of a velocity times Planck constant $\hbar .^{72-79} \omega_{p}(q), v_{p}$, and $m$ are, respectively, the dispersion relation of the phonon in state $(q, p)$, group velocity of a p-polarization phonon and directive cosine; cosine of the angle between the $x$ axis and the phonon wave-vector $\boldsymbol{q}$. We assume in our analysis the heat transport to be in the same direction as the applied temperature disturbance.

By using Callaway analysis and Debye-like phonon dispersion $\omega_{p}(q)=v_{p} q,{ }^{72-79}$ we can easily show that the term $g_{q, p}^{m}$ is given by

$$
g_{q, p}^{m}=-\beta_{p} \frac{\tau_{q, p}^{C}}{\tau_{q, p}^{N}} m v_{p} C_{q}^{p} \frac{d T}{d x} .
$$

$\beta_{p}$ is Callaway parameter that has the dimension of a relaxation time $^{72-79}$ and $C_{q}^{p}=\frac{\partial U_{q, p}^{0}}{\partial T}=\hbar \omega_{p} \frac{\partial n_{q, p}^{0}}{\partial T}$ is the specific heat or heat capacity per phonon normal mode. ${ }^{79}$ The Callaway pseudo-relaxation time $\beta_{\mathrm{p}}$ describing the effect of $\mathrm{N}$-processes is calculated as in the conventional steady-state local/linear treatment, by recalling that $\mathrm{N}$-processes cannot change the total phonon wave-vector (total crystal momentum). ${ }^{72-79}$
It is worthwhile mentioning here that the expression of the drift velocity $\frac{\lambda_{p}}{\hbar}$ of a phonon $(q, p)$ is proportional to $\frac{\left(\frac{d T}{d x}\right)}{T}$. Nevertheless, because of the smallness of Callaway pseudo-relaxation time $\beta_{p},{ }^{72,73}$ the proportionality constant is very small over almost the whole temperature range considered in our study. Therefore, we continue to suppose there is no need to use higher orders in the Taylor's expansion of $n_{q, p}^{\lambda_{P}}$ even in the case of very high values of $\frac{\left(\frac{d T}{d x}\right)}{T}$.

Equation (1) is a simple one variable inhomogeneous partial differential equation. Its solution can readily be obtained and we can easily show that we get the following solution:

$$
\begin{aligned}
U_{q, p}^{m}(x)= & \left(U_{q, p}^{0}+g_{q, p}^{m}\right)(x)+e^{-\frac{\xi_{q, p}(x)}{m}}\left[U_{q, p}^{m}(0)-\left(U_{q, p}^{0}+g_{q, p}^{m}\right)(0)\right] \\
& -\int_{0}^{x} d x^{\prime} e^{-\frac{\xi_{q, p}(x)-\xi_{q, p}\left(x^{\prime}\right)}{m}} \frac{d}{d x^{\prime}}\left[\left(U_{q, p}^{0}+g_{q, p}^{m}\right)\left(x^{\prime}\right)\right]
\end{aligned}
$$

where the function $\xi_{q, p}$ is defined as $\xi_{q, p}(x)=\int_{0}^{x} \frac{1}{\left(v_{p} \tau_{q, p}^{C}\right)} d x^{\prime}$.

A physically meaningful form of Eq. (3) is conditioned by the behavior of the exponential term that can grow to infinity when $x \rightarrow \pm \infty$ depending on the sign of the cosine $m$. Therefore, the boundary condition $U_{q, p}^{m}(0)$ plays a key role in the mathematical treatment. According to Mahan and Claro, $U_{q, p}^{m}(0)$ is selected to make the factor of the exponential term vanish at infinity ${ }^{48}$ and its exact form depends on the geometrical configuration: semi-infinite or infinite.

\section{B. Case of a semi-infinite geometry: TDTR and FDTR configurations}

The semi-infinite geometry of the SC crystal suits very well both TDTR and FDTR experimental configurations. $^{10,16,21,22,25,29,30,59,60,62,70}$ For these configurations, we will provide a theoretical framework to analyze the reduction of the effective thermal conductivity of a semi-infinite cubic SC crystal, at high frequencies of the oscillating heat source applied to its top surface. We assume the heating frequency to be much smaller than the average dominant phonon scattering rate over all branch polarizations. Hence, we neglect the time-scale effect and the steady-state form of the BPTE as given by Eq. (1) can describe the phonon transport process. In this case, $\xi_{q, p}$ represents the distance $x$ from the top surface normalized by the phonon MFP $l_{q, p}(x)=v_{p} \tau_{q, p}^{C}$.

As we shall see in Sec. III, this assumption is consistent with the previous assertion by Koh et al. ${ }^{10,60}$ The latter states that the reduction of the thermal conductivity of SC alloys in room temperature TDTR experiments at heating frequencies $<20 \mathrm{MHz}$ is primary an effect of length scales, i.e., mean free paths and thermal penetration depths and not an effect of time scales, i.e., phonon lifetimes and the period of the modulated heat source.

In this case, the SC crystal is assumed to be characterized by a fixed $U_{q, p}^{m}(0)$ at its top surface $(x=0)$ that we assume to be coated with a thin metallic layer at $(x<0)$ that plays the role of a heat transducer in TDTR and FDTR experiments. From this surface, heat will flow to the interior of the SC crystal along the $x$ axis. A very instructive illustration of the semi-infinite geometry applied to TDTR and FDTR configurations is reported in the paper of Koh 
et $a .^{60}$ As mentioned by Mahan and Claro ${ }^{48}$ and Koh et al., ${ }^{60}$ $U_{q, p}^{m}(0)$ may depend on many factors mainly related to the scattering, transmission, and reflection of phonons at this top boundary (junction between the metallic transducer and the SC substrate). We distinguish two classes of phonons; phonons that propagate to the surface $(m<0)$ and phonons that propagate from the surface $(m>0){ }^{60}$ Therefore, the analysis requires a special care in order to distinguish forward propagating phonons and "reflect" back-traveling phonons reaching the top surface back into the medium. For the backward phonons $(m<0)$, we follow Mahan and Claro ${ }^{48}$ and choose the expression of $U_{q, p}^{m<0}(0)$ that makes the factor of the exponential term $e^{-\frac{\xi_{q, p}(x)}{m}}$ in Eq. (3) tends to zero as $x \rightarrow+\infty$. Using the boundary condition $U_{q, p}^{m}(x \rightarrow+\infty)=0$ and $\left(U_{q, p}^{0}+g_{q, p}^{m}\right)(x \rightarrow+\infty)=0$, we obtain

$$
\begin{aligned}
U_{q, p}^{m<0}(0)= & \left(U_{q, p}^{0}+g_{q, p}^{m<0}\right)(0) \\
& +\int_{0}^{+\infty} d x^{\prime} e^{\frac{\xi_{q, p}\left(x^{\prime}\right)}{m}} \frac{d}{d x^{\prime}}\left[\left(U_{q, p}^{0}+g_{q, p}^{m<0}\right)\left(x^{\prime}\right)\right] .
\end{aligned}
$$

By inserting Eq. (4) into Eq. (3), we directly obtain the general solution for backward propagating phonons $(m<0)$ as

$$
\begin{aligned}
U_{q, p}^{m<0}(x)= & \left(U_{q, p}^{0}+g_{q, p}^{m<0}\right)(x) \\
& +\int_{x}^{+\infty} d x^{\prime} e^{-\frac{\xi_{q, p}(x)-\xi_{q, p}\left(x^{\prime}\right)}{m}} \frac{d}{d x^{\prime}}\left[\left(U_{q, p}^{0}+g_{q, p}^{m<0}\right)\left(x^{\prime}\right)\right] .
\end{aligned}
$$

To determine the expression of $U_{q, p}^{m>0}(0)$ for phonons that propagate away from the top surface $(m>0)$, we shall, in a rigorous treatment of the transient phonon transport regime, consider the scattering, transmission, and reflection of phonons at the junction between the metallic film transducer and the SC substrate that is responsible of the interface thermal resistance (ITR) at this junction. The ITR is undoubtedly one of the most fundamental elements in the study of heat transport in TDTR and FDTR experiments. ${ }^{1,2,21,59,65,70,80-82}$ Nonetheless, since we are considering only the steady-state regime, and although the phonon distribution at the interface (including both sides) might be in a nonequilibrium state, we could assume each side of this interface to be in local thermal equilibrium and as such is characterized by a constant temperature. We note $T_{S}$ the temperature for the SC side. Furthermore, we can treat the junction as an independent system, which will allow us decoupling the process of phonon transport within it from the intrinsic phonon transport process inside the SC substrate. As an independent system, the junction has a characteristic size that spans over both sides of the interface. In order to emphasize the analysis of the intrinsic phonon transport process inside the SC substrate, the condition $x=0$ corresponds to the part of the junction within the SC side. Therefore, at $x=0$, there is no phonon reflection, but instead a phonon exchange process that we assume to be elastic and can be treated independently for each phonon. Yet simple, this model is based on a realistic boundary condition that could be reasonably representative of the phonon physics occurring in the steady-state regime at the interface in TDTR and FDTR experiments. ${ }^{60}$
In such a scenario, the average number of phonons of frequency $\omega$ at $x=0$ (including both backward phonons with $m<0$ and forward phonons with $m>0$ ) is given by the Planck equilibrium distribution function, ${ }^{60}$

$$
\frac{1}{2}\left[U_{q, p}^{m<0}(0)+U_{q, p}^{m>0}(0)\right]=U_{q, p}^{0}(0) .
$$

By doing so, we can easily show that

$$
\begin{aligned}
U_{q, p}^{m>0}(0)= & \left(U_{q, p}^{0}+g_{q, p}^{m>0}\right)(0) \\
& -\int_{0}^{+\infty} d x^{\prime} e^{-\frac{\xi_{q, p}\left(x^{\prime}\right)}{m}} \frac{d}{d x^{\prime}}\left[\left(U_{q, p}^{0}-g_{q, p}^{m>0}\right)\left(x^{\prime}\right)\right] .
\end{aligned}
$$

Therefore, the general solution for forward propagating phonons $(m>0)$ is given by

$$
\begin{aligned}
U_{q, p}^{m>0}(x)= & \left(U_{q, p}^{0}+g_{q, p}^{m>0}\right)(x)-\int_{0}^{x} d x^{\prime} e^{-\frac{\xi_{q, p}(x)-\xi_{q, p}\left(x^{\prime}\right)}{m}} \frac{d}{d x^{\prime}}\left[\left(U_{q, p}^{0}+g_{q, p}^{m>0}\right)\left(x^{\prime}\right)\right] \\
& -\int_{0}^{+\infty} d x^{\prime} e^{-\frac{\xi_{q, p}(x)+\xi_{q, p}\left(x^{\prime}\right)}{m}} \frac{d}{d x^{\prime}}\left[\left(U_{q, p}^{0}-g_{q, p}^{m>0}\right)\left(x^{\prime}\right)\right]
\end{aligned}
$$

It is worth mentioning that the stringent boundary equilibrium condition on the SC side as given by Eq. (6) fulfills the energy conservation requirement $\frac{d J_{Q}}{d x}=0$ at $x=0$ where $J_{Q}$ is the heat flux density current along the direction $\vec{x}$ of the applied temperature disturbance. This condition may not be rigorously valid but can be justified by the final result that we shall discuss in Sec. III.

In the framework of Debye's model of the phonon dispersion relation, $J_{Q}$ is defined as $3,4,72,73$

$$
\begin{aligned}
J_{Q}(x) & =\frac{1}{4 \pi^{2}} \sum_{p} v_{p} \int_{0}^{q_{D}^{p}} q^{2}\left[\int_{-1}^{1} m U(x, m, q, p) d m\right] d q \\
& =\frac{1}{4 \pi^{2}} \sum_{p} v_{p} \int_{0}^{q_{D}^{p}} q^{2}\left[\int_{-1}^{0} m U_{q, p}^{m<0}(x) d m+\int_{0}^{1} m U_{q, p}^{m>0}(x) d m\right] d q,
\end{aligned}
$$

where $q_{D}^{P}$ denotes Debye's cutoff wave-vector of the acoustic branch polarization $p \cdot{ }^{3,4,72,73}$ The equilibrium phonon Planck distribution functions $n_{q, p}^{0}$ and $n_{q, p}^{E q}$ are symmetrical in the wave-vector $\boldsymbol{q}$ space while the group velocity is an algebraic function of $q$. The dispersion relation and the relaxation times depend on the module of the wave-vector $\boldsymbol{q}$ and as such are even functions of $q$. Therefore, the deviational equilibrium spectral energy density per phonon mode $U_{q, p}^{0}$ has no contribution to $J_{Q} \cdot{ }^{3,4,72,73}$ After some manipulations, 
we can straightforwardly write the expression of $J_{Q}(x)$ as

$$
\begin{aligned}
J_{Q}(x)= & \frac{1}{4 \pi^{2}} \sum_{p} v_{p} \int_{0}^{q_{D}^{p}} q^{2}\left\{\int_{-1}^{1} m g_{q, p}^{m}(x) d m\right. \\
& -\int_{0}^{1} m\left(\int_{x}^{+\infty} d x^{\prime} e^{\frac{\xi_{q, p}(x)-\xi_{q, p}\left(x^{\prime}\right)}{m}} \frac{d}{d x^{\prime}}\left[\left(U_{q, p}^{0}-g_{q, p}^{m}\right)\left(x^{\prime}\right)\right]\right) d m \\
& -\int_{0}^{1} m\left(\int_{0}^{x} d x^{\prime} e^{-\frac{\xi_{q, p}(x)-\xi_{q, p}\left(x^{\prime}\right)}{m}} \frac{d}{d x^{\prime}}\left[\left(U_{q, p}^{0}+g_{q, p}^{m}\right)\left(x^{\prime}\right)\right]\right) d m \\
& \left.-\int_{0}^{1} m\left(\int_{0}^{+\infty} d x^{\prime} e^{-\frac{\xi_{q, p}(x)+\xi_{q, p}\left(x^{\prime}\right)}{m}} \frac{d}{d x^{\prime}}\left[\left(U_{q, p}^{0}-g_{q, p}^{m}\right)\left(x^{\prime}\right)\right]\right) d m\right\} d q .
\end{aligned}
$$

By taking into account the expression of $g_{q, p}^{m}$ as given by Eq. (2) and the fact that $U_{q, p}^{0}$ depends on the position only through temperature $\frac{d U_{q, p}^{0}}{d x^{\prime}}=\left(\frac{d U_{q, p}^{0}}{d T}\right)\left(\frac{d T}{d x^{\prime}}\right)$, we easily identify the first term in Eq. (10) as describing the spatially local/linear contribution to the heat flux density current $J_{Q}$. On the other hand, the three other terms constitute the inherent spatially nonlocal/nonlinear contributions including two different driving potential forces; the conventional Fourier's temperature gradient $\frac{d T}{d x}$ and the temperature Laplacian $\frac{d^{2} T}{d x^{2}}$, respectively, as we shall see below.

Moreover, if one assumes small temperature perturbations at all locations of the SC crystal, the phonon relaxation times could be considered to be independent of $x$. Thus, $\xi_{q, p}(x)=\frac{x}{v_{p} \tau_{q, p}^{c}}=\frac{x}{l_{q, p}}$. The nonlinearity effects due to driving potential forces of the kind $(\nabla T)^{n}$ disappear and only remain the nonlocal effects. After some algebra and performing simple calculations, we obtain directly the final compact expression of $J_{Q}$ that we display in terms of the different driving potential forces as

$$
J_{Q}(x)=\left\{\begin{array}{c}
-\kappa_{\text {Loc }}^{N}(T) \frac{d T}{d x}-\int_{0}^{+\infty} d x^{\prime}\left[K_{1}^{\text {Nonloc }}\left(x-x^{\prime}\right)+K_{1}^{\text {Nonloc }}\left(x+x^{\prime}\right)\right] \frac{d T}{d x^{\prime}} \\
-\int_{x}^{+\infty} d x^{\prime} K_{2}^{\text {Nonloc }}\left(x-x^{\prime}\right) \frac{d^{2} T}{d x^{\prime 2}} \\
+\int_{0}^{x} d x^{\prime} K_{2}^{\text {Nonloc }}\left(x-x^{\prime}\right) \frac{d^{2} T}{d x^{\prime 2}}-\int_{0}^{+\infty} d x^{\prime} K_{2}^{\text {Nonloc }}\left(x+x^{\prime}\right) \frac{d^{2} T}{d x^{\prime 2}}
\end{array}\right\} .
$$

$\kappa_{L o c}^{N}$ denotes the spatially local/linear thermal conductivity due to the peculiar shuffling effect of the crystal momentum by phonon-phonon Normal scattering processes,

$$
\kappa_{L o c}^{N}(T)=\frac{1}{6 \pi^{2}} \sum_{p} \beta_{p} v_{p}^{2} \int_{0}^{q_{D}^{p}} C_{q}^{P} \frac{\tau_{q, p}^{C}}{\tau_{q, p}^{N}} q^{2} d q .
$$

We have also introduced two functions (thermal conduction kernels) $K_{1}^{\text {Nonloc }}$ and $K_{2}^{\text {Nonloc }}$ that are defined as

$$
\left\{\begin{array}{l}
K_{1}^{\text {Nonloc }}(x)=\frac{1}{4 \pi^{2}} \sum_{p} v_{p} \int_{0}^{q_{D}^{p}} C_{q}^{P} q^{2} E_{3}\left(\frac{|x|}{v_{p} \tau_{q, p}^{C}}\right) d q \\
K_{2}^{\text {Nonloc }}(x)=\frac{1}{4 \pi^{2}} \sum_{p} \beta_{p} v_{p}^{2} \int_{0}^{q_{D}^{p}} C_{q}^{P} \frac{\tau_{q, p}^{C}}{\tau_{q, p}^{N}} q^{2} E_{4}\left(\frac{|x|}{v_{p} \tau_{q, p}^{C}}\right) d q
\end{array}\right.
$$

where $E_{v}(z)=\int_{0}^{1} m^{v-2} e^{-\frac{z}{m}} d m$ is the exponential integral function of order $v$.

\section{RESULTS AND DISCUSSION}

In the current analysis, we seek to study the relevance and implications of nonlocal effects on the steady-state onedimensional transport behavior of the phonon gas in a region of a semi-infinite SC crystal subject to an external heat source applied to its top surface, giving rise to different heat driving potential forces.

In Sec. II, we made the assumption that the Callaway pseudo-relaxation time $\beta p$, describing the implicit effect of $\mathrm{N}$-processes, does not depend on space and that this approximation should preserve the essential features of thermal conduction by phonons. This means that the dependence of the phonon gas drift on space is contained in the expression of the drift velocity $\frac{\lambda_{p}}{\hbar}$ and thus in the one of $g_{q, p}^{m}$, only through the temperature gradient $\frac{d T}{d x} \cdot{ }^{73}$ $\beta p$ is a complicated quantity, depending on $\tau_{q, S}^{N}$ and $\tau_{q, S}^{R}$. This complication is necessary because of the behavior of $\mathrm{N}$-processes, which shuffle crystal momentum back and forth between normal modes, and then contribute implicitly to the lattice thermal conduction (resistance) process of a given SC crystal material. ${ }^{72,73}$

As one can see in Eq. (11), the peculiar implicit shuffling effect of the crystal momentum by phonon-phonon Normal scattering processes plays a key and central role in the onset of thermal conduction. Taking into account the inherent spatial nonlocality effects tends to accentuate the role of phonon-phonon scattering $\mathrm{N}$-processes, as the latter are at the origin of existence of the temperature Laplacian as a second driving potential force for the heat flux density current in addition to the conventional Fourier's temperature gradient. A similar feature has been shown in the past in the seminal work of Guyer and Krumhansl. ${ }^{83}$

A first trivial result that we can issue from the theory developed above deals with the effect of a constant temperature gradient 
on the expression of the heat flux density current. In this particular case, the temperature Laplacian vanishes and all nonlocality effects disappear; the process of phonon heat transport becomes totally local in a Fourier's sense. Indeed, we can easily show that the integral of the first thermal conduction kernel $K_{1}^{\text {Nonloc }}$ in Eq. (11) becomes a local quantity independent of $x$, and we obtain an effective thermal conductivity given exactly by the expression of the conventional Fourier's thermal conductivity here and henceforth noted $\kappa_{\text {Four }}^{C}{ }^{72-79}$

$$
\kappa_{\text {Four }}^{C}(T)=\frac{1}{6 \pi^{2}} \sum_{p} v_{p}^{2} \int_{0}^{q_{D}^{p}} C_{q}^{P} \tau_{q, p}^{C}\left(1+\frac{\beta_{p}}{\tau_{q, p}^{N}}\right) q^{2} d q .
$$

\section{A. Phonon transport in TDTR and FDTR configurations}

TDTR and FDTR techniques constitute the first set of experimental configurations that have been used to investigate nondiffusive phonon transport in dielectric and SC crystal materials. $^{10,16,21,22,25,29,30,59,60,62,70}$ One of the pioneer works is Koh and Cahill's back in 2007. ${ }^{10}$ By varying the frequency of modulation of the pump beam in their TDTR experiment, Koh and Cahill explored the onset of quasi-ballistic phonon transport in SC crystals. Indeed, the measured apparent or effective thermal conductivity $\kappa_{E f f}$ of the SC crystals they studied has shown a monotonic decrease as a function of this frequency. Over the frequency range used in their experiments $(0.6-10 \mathrm{MHz})$, the results show $\kappa_{E f f}$ of SC alloys to undergo a substantial decrease while, for single SC crystals, $\kappa_{E f f}$ manifested a plateau at room temperature. In the diffusive regime, a characteristic length of the temperature profile that plays a key role in this configuration is the thermal penetration depth as defined based on Fourier's law $\mu=\sqrt{\frac{\alpha}{\pi F}}$, where $\alpha$ is the thermal diffusivity and $F$ is the frequency of the applied oscillating heat source. $\mu$ characterizes the distance over which heat can diffuse in one period of oscillation. At fixed $T$ and depending on $F$, $\mu$ can indeed be shorter than the dominant phonon MFP. ${ }^{10}$

Since then, few theoretical approaches have been suggested in order to explain the experimental observations. In their first interpretation of the results, Koh and Cahill used the BPTE in the steady-state regime with the modified Debye-Callaway model in which they assumed a boundary scattering process that phonons would undergo at a virtual interface. This virtual interface is actually the surface of a hemisphere whose radius is the thermal penetration depth $\mu .{ }^{10}$ The authors found a good and satisfactory agreement between experimental data and the results of this phenomenological approach. Da Cruz et al. suggested that the reduction in the measured apparent thermal conductivity could instead be due to the insensitivity of TDTR experiments to phonons with $l_{\text {anh }}>3 \mu$, where $l_{\text {anh }}$ is the MFP of phonons due to anharmonic scattering only. ${ }^{18}$ Their calculations using the fitted cutoff of $3 \mu$ agreed well with the frequency-dependent TDTR measurements. ${ }^{18}$ A "two-fluid" or "two-temperature" model approach based on conventional Fourier's heat diffusion equation has also been presented by Wilson et al. ${ }^{21}$ In this approach, phonons are divided into two subsystems, low-frequency phonons and high frequency phonons, characterized by two different temperatures and interacting through a coupling parameter. This approach was also capable of explaining satisfactorily the experimental observations in terms of an interfacial nonequilibrium conductance. ${ }^{21}$ Wilson and Cahill also developed a ballistic/diffusive nonlocal model that kept the same guidelines of the "two-temperature" model but added more details in analyzing the reduced apparent cross-plane thermal conductivity of SC alloys as essentially due to an inhomogeneous effective thermal conductivity near the top surface of the samples that is caused by the transmission and reflection of long MFP phonon at the interface between the metal transducer and the SC substrate. ${ }^{59}$ Later, Koh et al. ${ }^{60}$ used the Mahan and Claro nonlocal theory ${ }^{48}$ to build a more robust framework and by doing so, the authors have brought more elements to explain their experimental observations regarding the frequency dependence of the measured $\kappa_{E f f}$ in TDTR experiments. Yang and Dames used a two-flux model to solve the conventional single mode relaxation time approximated BPTE without Callaway's collision operator (standard relaxation time approximation, RTA). ${ }^{32}$ They obtained an analytical solution for the suppression function of $\kappa_{E f f}$ in the gray spectrum approximation. ${ }^{32}$ Another approach explored by Vermeersch et al. took a completely different path as it used Lévy flights random walk formalism. ${ }^{61,62,71}$ The latter formalism turns out to be very successful in the interpretation of the experimental results of TDTR experiments. ${ }^{62}$ Last but not least is the work of Hua et al., who presented recently a very interesting and detailed model based on the standard RTA of the BPTE in the transient regime, in which they derived a generalized Fourier's law to study non-diffusive thermal transport in general geometries. ${ }^{70}$ Besides the inherent nonlocality, the model includes inhomogeneous nonlocal terms arising from the heat source or the boundary conditions. The model looks very robust and allowed obtaining a very good agreement between the theoretical predictions and TDTR thermal responses. ${ }^{70}$

In the present work, we show that application of the nonlocal theory of Mahan and Claro ${ }^{48}$ to the steady-state single mode relaxation time approximated BPTE using Callaway's collision operator allows indeed obtaining an analytical expression of the apparent or effective thermal conductivity $\kappa_{E f f}$ that would be measured in TDTR and FDTR experiments. The integrand of $\kappa_{E f f}$ includes a spectral suppression function which captures elegantly how the frequency of the applied oscillating heat source at the top surface of a semi-infinite SC crystal intervenes in the onset of the quasi-ballistic heat transport regime. The relation between the spectral phonon MFP and the thermal penetration depth $\mu$ conditions this regime, as we shall see next.

In a TDTR or FDTR experiment, the sample is heated at the surface and the thermal response of the sample is measured at the same surface and used to determine the thermal properties. ${ }^{10,60}$ When a semi-infinite solid is subjected to a periodic heat source of the form $P e^{-2 \pi F t}$ where $P$ is the power per unit area, it is very straightforward to show that the generated oscillating temperature field within it is given by

$$
T(x, t)=\frac{P}{\sqrt{\kappa C_{V} 2 \pi F}} e^{-\frac{x}{\mu}}\left(\frac{1+i}{\sqrt{2}}\right) e^{-i\left(2 \pi F t-\frac{x}{\mu}\right)},
$$

where $\varepsilon=\sqrt{\kappa C_{V}}$ denotes the thermal effusivity that we assume to include the contribution from all phonons, diffusive and non- 
diffusive (quasi-ballistic), and as such does not depend on the applied modulation frequency in the experiment. ${ }^{10}$

In order to extract the expression of the apparent or effective thermal conductivity $\kappa_{E f f}$ that would be measured in TDTR and FDTR experiments, we consider the exponentially decaying envelop or amplitude of the oscillating temperature field of Eq. (15) given by $T_{S} e^{-\frac{x}{\mu}}$ where $T_{S}=\frac{P}{\varepsilon \sqrt{2 \pi F}}$ designates the magnitude of $T$ at $x=0$ as we mentioned earlier. We assume this envelop to contain sufficient information to provide insights into the frequency dependence of $\kappa_{E f f}$ measured in these experiments. It is important to note however that the frequency dependence of the temperature and the heat flux density current at the surface of the semi-infinite SC crystal does not conflict with the steady-state assumption we made in Sec. II regarding these two quantities as long as the applied modulation frequency in the experiment is much lower than the average dominant phonon scattering rate over all branch polarizations.

We inject this expression of the temperature profile into the expression of the heat flux density current calculated for the 1D semi-infinite geometry as given by Eq. (11). It is very straightforward to carry out all different integral calculations involved in this equation. We perform the integration calculations in two steps: first, with respect to the space variable $x^{\prime}$, we take the result at $x=0$ and then we integrate with respect to the directive cosine $m$. An alternative way is to use directly the definition of $J_{Q}$ at $x=0$ in combination with the expressions of $U_{q, p}^{m<0}(0)$ and $U_{q, p}^{m>0}(0)$ as given by Eqs. (4) and (7), respectively.

We define $\kappa_{E f f}$ using Fourier's law expressed at the top surface $(x=0)$ as

$$
\left.J_{Q}(0, T)=-\kappa_{E f f} \frac{d T}{d x}\right)_{x=0}=\frac{T_{S}}{\mu} \kappa_{E f f} .
$$

After some mathematical manipulations, we get directly the final compact expression of $\kappa_{E f f}$ as

$$
\left\{\begin{array}{l}
\kappa_{E f f}(T, F)=\frac{1}{2 \pi^{2}} \sum_{p} v_{p}^{2} \int_{0}^{q_{D}^{p}} C_{q}^{P} \tau_{q, p}^{C}\left(1+\frac{\beta_{p}}{\tau_{q, p}^{N}}\right) \Xi\left(\frac{v_{p} \tau_{q, p}^{C}}{\mu}\right) q^{2} d q \\
\Xi(z)=\frac{1}{2 z}-\frac{1}{z^{2}}+\frac{\log [1+z]}{z^{3}} ; z=\frac{v_{p} \tau_{q, p}^{C}}{\mu} \text { and } \mu(F)=\sqrt{\frac{\alpha}{\pi F}}
\end{array} .\right.
$$

$\Xi$ is the spectral suppression function in TDTR and FDTR configurations that allows quantifying the reduction of the thermal conductivity as a function of the modulation frequency $F$. It is worth mentioning that we can obtain the same spectral suppression function using the standard RTA of the BPTE and following the same mathematical steps and assumptions as above. In this case, $J_{Q}$ will be given by solely the second term of Eq. (11). All other terms of Eq. (11) related to Callaway's parameter $g_{q, p}^{m}$ vanish as they represent the manifestation of the peculiar effects of phonon-phonon $\mathrm{N}$-processes that distinguish them from the rest of phonon scattering processes including phonon-phonon U-processes. The standard RTA usually used does not take this difference into consideration, which can be justified at high temperatures where
$\mathrm{U}$-processes dominate $\mathrm{N}$-processes, but not in the low temperature regime. ${ }^{3,4,72,73}$

The first remarkable feature of $\Xi$ is its universal character as it depends only on the ratio of the phonon MFP and the thermal penetration depth $\mu$ that is the characteristic length scale in this case. $\mu$ as defined based on the diffusive Fourier's law appears naturally in the calculation once an analogy with Fourier's based thermal conductivity is sought. $\Xi$ is a monotonically increasing (decreasing) function of thermal penetration depth (nondimensional parameter $z$ ). It is easy to check under the condition of constant $\alpha$ that $\operatorname{Lim} \Xi(T, F)=\frac{1}{3}$ so that $\kappa_{E f f}$ will tend exactly to the expression of the conventional Fourier's thermal conductivity $\kappa_{\text {Four }}^{C}$ [Eq. (14)] of a bulk SC crystal in the framework of the modified Debye-Callaway model. ${ }^{78,79}$ On the other hand, $\underset{F \rightarrow+\infty}{\operatorname{Lim}} \Xi(T, F)=0$ and $\kappa_{E f f}$ will tend ultimately to zero; in the very high frequency regime, the SC crystal becomes completely insulating to phonon transport and no thermal conduction process can take place. Indeed, in the regime of high frequencies, $\kappa_{E f f}$ decreases according to $\frac{1}{F}$ power law.

It is very interesting to note that even though the high frequency regime (modulation frequencies much higher than the average dominant phonon scattering rate over all branch polarizations) is out of the domain of validity of the assumptions mentioned in the theoretical approach as presented above in Sec. II, the limiting behavior of $\Xi$ in this regime leads to the correct and sound physical result for $\kappa_{E f f}$. Nevertheless, while the limiting behavior will be the same, the real asymptotic behavior of $\kappa_{E f f}$ as a function of the modulation frequency in the high frequency regime will necessitate solving the full transient BPTE including both time and space derivatives which is out of the scope of the present work.

The true advantage of this spectral suppression function is that on the one hand, it generalizes the results of Yang and Dames ${ }^{32}$ and on the other hand, it constitutes a direct tool that gives more analysis freedom and easiness for the interpretation of the experimental reduction of $\kappa_{E f f}$ as a function of the modulation frequency in TDTR and FDTR configurations. Indeed, the utilization of this spectral suppression function does not demand any costly numerical computations to calculate the full thermal response as it is the case in some of the other developed approaches. ${ }^{59,60,70}$

We consider bulk $\mathrm{Si}$ and $\mathrm{Si}_{0.7} \mathrm{Ge}_{0.3}$ cubic SC crystals to illustrate the steady-state behavior of $\kappa_{E f f}$ as a function of temperature and modulation frequency. $\mathrm{Si}$ and $\mathrm{Si}_{0.7} \mathrm{Ge}_{0.3}$ are representative of single and alloy SC crystals, respectively. In addition to three phonon-phonon anharmonic scattering $\mathrm{U}$ and $\mathrm{N}$ processes, we assume scattering of phonons by the boundaries of the bulk SC crystal and by impurities (defects). The expression of the relaxation times of the different phonon scattering processes considered as well as the geometrical and physical properties of $\mathrm{Si}$ and $\mathrm{Si}_{0.7} \mathrm{Ge}_{0.3}$ materials can be found in Tables I and II of Ref. 79. We assume all physical properties of these SC crystals to be independent of temperature. The total resistive phonon scattering rate $\left(\tau_{q, p}^{R}\right)^{-1}$ is obtained via Matthiessen's rule. ${ }^{77-79}$

In order to simplify more the expression of $\kappa_{E f f}$ in Eq. (17), we express it, as it is customary in the modified Debye-Callaway model, as the sum over one longitudinal $\left(\kappa_{E f f}^{L}\right)$ and two degenerate transverse $\left(\kappa_{E f f}^{T}\right)$ phonon acoustic branch polarizations. ${ }^{77-79} \mathrm{We}$ 


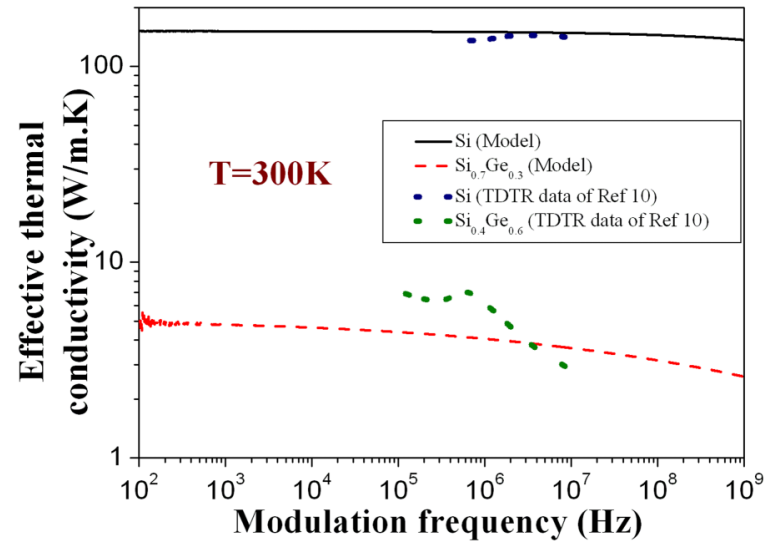

FIG. 1. Computed steady-state room temperature behavior of the effective thermal conductivity of $\mathrm{Si}$ and $\mathrm{Si}_{0.7} \mathrm{Ge}_{0.3}$ bulk $\mathrm{SC}$ crystals in TDTR and FDTR experiments as a function of the modulation frequency.

also make use of the usual change of variable $y=\frac{\hbar \omega}{k_{B} T}$, which allows us to obtain straightforwardly the final workable and computational expression of $\kappa_{E f f}$.

Figure 1 reports the computed steady-state behavior of $\kappa_{E f f}$ of bulk $\mathrm{Si}$ and $\mathrm{Si}_{0.7} \mathrm{Ge}_{0.3}$ cubic SC crystals at room temperature as a function of the modulation frequency in TDTR and FDTR experiments. At $F=10 \mathrm{MHz}, \kappa_{E f f}$ of bulk Si decreases by barely $2 \%$ with regard to its value at very low frequency. The reduction rate reaches $10 \%$ at $F=1 \mathrm{GHz}$. On the other hand, $\kappa_{E f f}$ of $\mathrm{Si}_{0.7} \mathrm{Ge}_{0.3}$ decreases significantly with frequency. At $F=10 \mathrm{MHz}$, it decreases by almost $27 \%$ with regard to its value at very low frequency. The reduction rate reaches even $48 \%$ at $F=1 \mathrm{GHz}$. We report on the same figure the experimental TDTR data of Koh and Cahill for the room temperature effective thermal conductivity of $\mathrm{Si}$ and $\mathrm{Si}_{0.4} \mathrm{Ge}_{0.6}$ in order to illustrate the relevance of the theoretical prediction. Even though the experimental data span over a small frequency interval, we can clearly observe that the computed modulation frequency behavior of $\kappa_{E f f}$ for both $\mathrm{Si}$ and $\mathrm{Si}_{0.7} \mathrm{Ge}_{0.3}$ reproduces quite fairly the experimental observations of Koh and Cahill for SC single crystals and alloys, respectively. ${ }^{10} \mathrm{Si}_{0.4} \mathrm{Ge}_{0.6}$ alloy has indeed a higher thermal conductivity than $\mathrm{Si}_{0.7} \mathrm{Ge}_{0.3}$. In addition, its effective thermal conductivity appears to show a higher decreasing rate as a function of the TDTR modulation frequency.

One key parameter in obtaining the computed graphs in this figure is the value of the thermal diffusivity $\alpha$ used to calculate the thermal penetration depth $\mu$. For sake of simplicity, we used the conventional Fourier's value of $\alpha$ giving by $\alpha_{\text {Four }}^{C}=\frac{\kappa_{\text {Four }}^{C}}{C_{V}}$ where $\kappa_{\text {Four }}^{C}$ is the conventional Fourier's thermal conductivity and $C_{V}$ designates the heat capacity per unit volume calculated by taking into account only acoustic phonon branches and polarizations as it is the case for $\kappa_{\text {Four }}^{C}{ }^{79}$ The steady-state temperature behavior of $\alpha$ for both $\mathrm{Si}$ and $\mathrm{Si}_{0.7} \mathrm{Ge}_{0.3}$ is reported in Fig. 2 .

The utilization of this $\alpha$ might raise a consistency question regarding the intertwining dependency between thermal diffusivity and conductivity. Meanwhile, it is the most straightforward way to

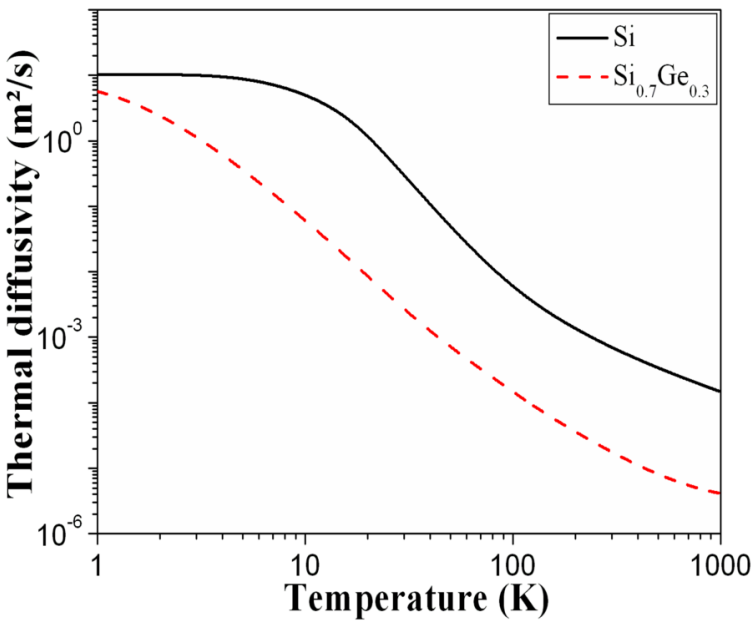

FIG. 2. Computed steady-state behavior of the conventional Fourier's thermal diffusivity of $\mathrm{Si}$ and $\mathrm{Si}_{0.7} \mathrm{Ge}_{0.3}$ bulk $\mathrm{SC}$ crystals as a function of temperature.

give an insight into the modulation frequency behavior of $\kappa_{E f f}$ without major numerical iterative integral computations that surely are not necessary regarding the simplification assumptions used in the theoretical approach in the first place.

Besides, consideration of the contribution of optical phonons to $C_{V}$ will decrease $\alpha$ and increasing $F$ leads to a reduction in $\alpha$ as well. According to the expression of $\mu$, increasing $F$ or decreasing $\alpha$ have the same action on $\mu$. Therefore, the decreasing rate of $\kappa_{E f f}$ as a function of $F$ is expected to be higher due to an accumulation or reinforcement effect, but the general behavior will remain the same as the one reported above in Fig. 1.

The effect of changing the ambient temperature on the modulation frequency behavior of $\kappa_{E f f}$ is shown in Figs. 3(a) and 3(b) for bulk $\mathrm{Si}$ and $\mathrm{Si}_{0.7} \mathrm{Ge}_{0.3}$ cubic SC crystals, respectively. The decreasing rate of $\kappa_{E f f}$ for both SC crystals tends to increase by decreasing $T$. The effect of the modulation frequency on the behavior of the apparent $\kappa_{E f f}$ is thus highly dependent on the ambient temperature. Indeed, the latter directly influences the phonon relaxation time and therefore conditions the relation between the phonon MFP and the thermal penetration depth, which is the characteristic length in TDTR and FDTR configurations as we mentioned earlier. We assumed the validity of the steady-state regime theoretical approach to hold down to $3 \mathrm{~K}$. Of course, this could be questionable since the average phonon relaxation time tends to increase by decreasing $T$. ${ }^{3,4}$ Nevertheless, the general trend will still remain correct to a certain frequency threshold.

TDTR/FDTR and TTG are two different but complementary experimental techniques in a sense that the former one is more sensitive to the cross-plane phonon transport while the latter is more sensitive to the in-plane one. These two techniques have different excitation and detection procedures. Therefore, it is not surprising that the spectral suppression function $\Xi$ of $\kappa_{E f f}$ we obtained herein for TDTR and FDTR configurations as given by Eq. (17) has a different expression than the one obtained for the TTG 

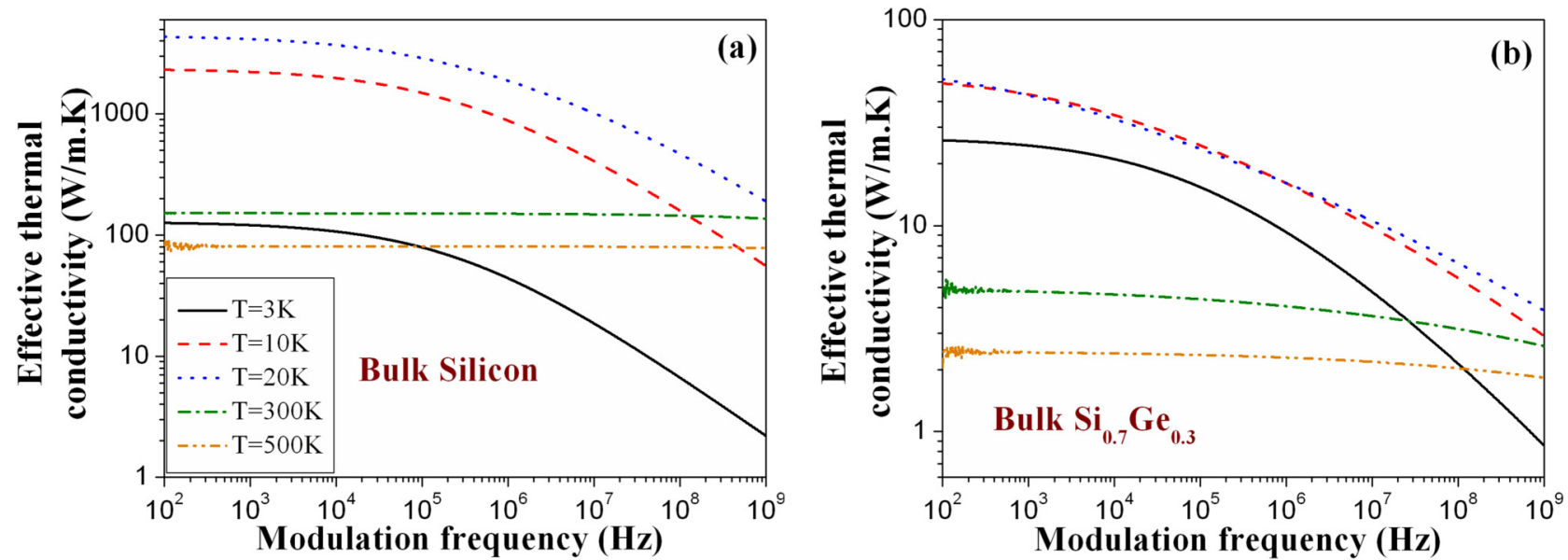

FIG. 3. Computed steady-state behaviors of the effective thermal conductivity of $\mathrm{Si}$ (a) and $\mathrm{Si}_{0.7} \mathrm{Ge}_{0.3}$ (b) bulk $\mathrm{SC}$ crystals in TDTR and FDTR experiments as functions of the modulation frequency for different temperatures.

configuration. $^{14,27,39} \Xi$ plays in TDTR and FDTR configurations the same role it does in the TTG configuration as it captures with great perspicacity the intrinsic intertwining interaction between low and high frequency phonons in the onset of quasi-ballistic and non-diffusive heat transport regime in SC crystals with the thermal penetration depth being the central characteristic length scale. All the tools for phonon spectroscopy including the accumulation function and the thermal conductivity per phonon MFP can be applied and therefore the phonon MFP distribution spectrum can be obtained in a similar manner as for the TTG configuration using an adapted reconstruction method. ${ }^{17,32}$ It is important to emphasize that the thermal penetration depth as defined based on the diffusive Fourier's law appears naturally in the calculation once an analogy with Fourier's based thermal conductivity is sought. On the other hand, and because of the nature of TTG experimental excitation and detection, no Fourier's based thermal penetration would appear in the calculation, but instead the optical penetration depth could have an effect as mentioned by Huberman et $a l .{ }^{45}$ In a TTG configuration, it is rather the thermal grating period that constitutes the central characteristic length scale. ${ }^{14,17,20,27,31,35,39,41,45}$

A cornerstone hypothesis we used in our modeling is related to the possibility of decoupling phonon transport process at the interface within the junction between the metallic film transducer and the SC substrate from the intrinsic phonon transport process inside the latter. We assumed this hypothesis to be fully justified in the steady-state regime. The ability to obtain a universal spectral suppression function that only depends on the ratio of the phonon MFP to the thermal penetration depth and that is capable of interpreting the reduction of $\kappa_{E f f}$ as a function of the modulation frequency in TDTR and FDTR techniques is a powerful and robust argument to support the validity of our fundamental hypothesis.

Meanwhile, a rigorous treatment of the transient phonon transport regime shall ineluctably consider the scattering, transmission, and reflection of phonons at the interface between the metallic film transducer and the SC substrate that is responsible of the
ITR at this junction. In this case, the phonon transport processes within both the metal film transducer and the SC substrate are coupled through the inherent phonon scattering processes within the junction between them which, in addition, lead to a highly nonequilibrium phonon distribution within this junction. In this case, the phonon transmission coefficients will undoubtedly be crucial. ${ }^{65,70}$ This situation would occur in the regime of high modulation frequencies or even potentially in the low temperature regime.

The next step of the study consists in generalizing the theory developed above to the $3 \mathrm{D}$ configuration in order to analyze the effect of the finite geometry of the pump laser spot in TDTR and FDTR experiments as well as to explore the case of anisotropic thermal conduction. This work is under way, and the main results will be available in a near future.

\section{SUMMARY}

An approach based on solving steady-state single mode relaxation time approximated Boltzmann-Peierls transport equation (BPTE) by application of the nonlocal theory of Mahan and Claro within the framework of the modified Debye-Callaway model has been developed to analyze the steady-state phonon heat transport in a semi-infinite cubic semiconductor (SC) crystal. A realistic boundary condition is assigned at the top surface of the SC crystal that can be considered reasonably representative of time domain thermoreflectance (TDTR) and frequency domain thermoreflectance (FDTR) experiments. We have obtained an expression of the heat flux density current that undoubtedly shed light on the crucial and relevant role of nonlocal spatial effects in steady-state phonon heat transport in SC crystals. The meticulously implicit shuffling effect of the crystal momentum by phonon-phonon Normal scattering processes plays a key and central role in the onset of thermal conduction. Taking into account the inherent spatial nonlocality effects tends to accentuate the role of phonon-phonon scattering 
$\mathrm{N}$-processes, as the latter introduce the temperature Laplacian as a second driving potential force for the heat flux density current in addition to the conventional Fourier's temperature gradient. We have derived a formula of the effective thermal conductivity of the SC crystal that is characterized with a spectral suppression function that captures and describes with great perspicacity the intertwining interaction between low and high frequency phonons in the onset of quasi-ballistic and non-diffusive heat transport regime. This spectral suppression function constitutes a direct tool that gives more analysis freedom and easiness for the interpretation of the experimental reduction of the effective thermal conductivity $\kappa_{E f f}$ as a function of the modulation frequency in TDTR and FDTR techniques. Indeed, its utilization does not demand any costly numerical computations to calculate the full thermal response as it is the case in some of the other developed approaches and its application covers all temperature range. The spectral suppression function is a universal function that only depends on the ratio between the phonon MFP and the thermal penetration depth as defined based on the diffusive Fourier's law and that is the central characteristic length scale in TDTR and FDTR configurations. Besides, all the available tools for phonon spectroscopy including the accumulation function and the thermal conductivity per phonon MFP can be applied and, hence, the phonon MFP distribution spectrum can be obtained in a similar manner as for the Transient Thermal Grating technique using an adapted reconstruction method. This analysis will therefore allow a better understanding of how thermal length scales affect which phonons conduct heat in each transport regime in TDTR and FDTR configurations.

\section{DATA AVAILABILITY}

The data that support the findings of this study are available within the article.

\section{REFERENCES}

${ }^{1}$ D. G. Cahill, W. K. Ford, K. E. Goodson, G. D. Mahan, A. Majumdar, H. J. Maris, R. Merlin, and S. R. Phillpot, J. Appl. Phys. 93, 793 (2003).

${ }^{2}$ D. G. Cahill, P. V. Braun, G. Chen, D. R. Clarke, S. Fan, K. E. Goodson, P. Keblinski, W. P. King, G. D. Mahan, A. Majumdar, H. J. Maris, S. R. Phillpot, E. Pop, and L. Shi, Appl. Phys. Rev. 1, 011305 (2014).

${ }^{3}$ J. M. Ziman, Electron and Phonons (Oxford University Press, New York, NY, 1960).

${ }^{4}$ G. P. Srivastava, The Physics of Phonons (Adam Hilger, Bristol, 1990).

${ }^{5}$ A. Henry and G. Chen, J. Comput. Theor. Nanosci. 5, 141 (2008).

${ }^{6}$ L. Lindsay, D. A. Broido, and T. L. Reinecke, Phys. Rev. B 87, 165201 (2013).

${ }^{7}$ D. D. Joseph and L. Preziosi, Rev. Mod. Phys. 61, 41 (1989).

${ }^{8}$ A. A. Joshi and A. Majumdar, J. Appl. Phys. 74, 31 (1993).

${ }^{\mathbf{9}}$ G. Chen, Phys. Rev. Lett. 86, 2297 (2001).

${ }^{10}$ Y. K. Koh and D. G. Cahill, Phys. Rev. B 76, 075207 (2007).

${ }^{11}$ Y. Ezzahri and A. Shakouri, Phys. Rev. B 79, 184303 (2009).

${ }^{12}$ M. E. Siemens, Q. Li, R. Yang, K. A. Nelson, E. H. Anderson, M. M. Murnane, and H. C. Kapteyn, Nat. Mater. 9, 26 (2010).

${ }^{13}$ Y. Ezzahri, K. Joulain, and A. Shakouri, J. Heat Transfer 133, 072401 (2011).

${ }^{14}$ A. A. Maznev, J. A. Johnson, and K. A. Nelson, Phys. Rev. B 84, 195206 (2011).

${ }^{15}$ A. J. Minnich, G. Chen, S. Mansoor, and B. S. Yilbas, Phys. Rev. B 84, 235207 (2011).

${ }^{16}$ A. J. Minnich, J. A. Johnson, A. J. Schmidt, K. Esfarjani, M. S. Dresselhaus,

K. A. Nelson, and G. Chen, Phys. Rev. Lett. 107, 095901 (2011).
${ }^{17}$ A. J. Minnich, Phys. Rev. Lett. 109, 205901 (2012).

${ }^{18}$ C. A. Da Cruz, W. Li, N. A. Katcho, and N. Mingo, Appl. Phys. Lett. 101, 083108 (2012).

${ }^{19}$ M. Maldovan, Appl. Phys. Lett. 101, 113110 (2012).

${ }^{20}$ J. A. Johnson et al., Phys. Rev. Lett. 110, 025901 (2013).

${ }^{21}$ R. B. Wilson, J. P. Feser, G. T. Hohensee, and D. G. Cahill, Phys. Rev. B 88, 144305 (2013).

${ }^{22}$ J. P. Freedman, J. H. Leach, E. A. Preble, Z. Sitar, R. F. Davis, and J. A. Malen, Sci. Rep. 3, 2963 (2013).

${ }^{23}$ K. C. Collins, A. A. Maznev, Z. Tian, K. Esfarjani, K. A. Nelson, and G. Chen, J. Appl. Phys. 114, 104302 (2013).

${ }^{24}$ A. A. Maznev, J. Appl. Phys. 113, 113511 (2013).

${ }^{25}$ K. T. Regner, D. P. Sellan, Z. Su, C. H. Amon, A. J. H. McGaughey, and J. A. Malen, Nat. Commun. 4, 1640 (2013).

${ }^{\mathbf{2 6}}$ K. T. Regner, A. J. H. McGaughey, and J. A. Malen, Phys. Rev. B 90, 064302 (2014).

${ }^{27}$ C. Hua and A. J. Minnich, Phys. Rev. B 89, 094302 (2014).

${ }^{28}$ C. Hua and A. J. Minnich, Phys. Rev. B 90, 214306 (2014).

${ }^{29} \mathrm{Y}$. Hu, L. Zeng, A. J. Minnich, M. S. Dreselhaus, and G. Chen, Nat. Nanotechnol. 10, 701 (2015).

${ }^{30}$ L. Zeng, K. C. Collins, Y. Hu, M. N. Luckyanova, A. A. Maznev, S. Huberman, V. Chiloyan, J. Zhou, X. Huang, K. A. Nelson, and G. Chen, Sci. Rep. 5, 17131 (2015).

${ }^{31}$ J. Cuffe, J. K. Eliason, A. A. Maznev, K. C. Collins, J. A. Johnson, A. Shchepetov, M. Prunnila, J. Ahopelto, C. M. Sotomayor Torres, G. Chen, and K. A. Nelson, Phys. Rev. B 91, 245423 (2015).

${ }^{32}$ F. Yang and C. Dames, Phys. Rev. B 91, 165311 (2015).

${ }^{33}$ A. J. Minnich, Phys. Rev. B 92, 085203 (2015).

${ }^{34}$ A. J. Minnich, Appl. Phys. Lett. 107, 183106 (2015).

${ }^{35}$ J. A. Johnson, J. K. Eliason, A. A. Maznev, T. Luo, and K. A. Nelson, J. Appl. Phys. 118, 155104 (2015).

36. Maassen and M. Lundstrom, J. Appl. Phys. 117, 135102 (2015).

37. Maassen and M. Lundstrom, J. Appl. Phys. 117, 035104 (2015).

${ }^{38}$ A. T. Ramu and J. E. Bowers, Appl. Phys. Lett. 106, 263102 (2015).

${ }^{39}$ V. Chiloyan, L. Zeng, S. Huberman, A. A. Maznev, K. A. Nelson, and G. Chen, Phys. Rev. B 93, 155201 (2016).

${ }^{40}$ L. Zeng, V. Chiloyan, S. Huberman, A. A. Maznev, J.-P. M. Peraud, N. G. Hadjiconstantinou, K. A. Nelson, and G. Chen, Appl. Phys. Lett. 108, 063107 (2016).

${ }^{41}$ A. Vega-Flick, R. A. Duncan, J. K. Eliason, J. Cuffe, J. A. Johnson, J.-P. M. Peraud, L. Zeng, Z. Lu, A. A. Maznev, E. N. Wang, J. J. Alvarado-Gil, M. Sledzinska, C. M. Sotomayor Torres, G. Chen, and K. A. Nelson, AIP Adv. 6, 121903 (2016).

42 J. Maassen and M. Lundstrom, J. Appl. Phys. 119, 095102 (2016).

${ }^{43}$ V. Chiloyan, L. Zeng, S. Huberman, A. A. Maznev, K. A. Nelson, and G. Chen, J. Appl. Phys. 120, 025103 (2016).

${ }^{44}$ M. Forghani, N. G. Hadjiconstantinou, and J.-P. M. Péraud, Phys. Rev. B 94, 155439 (2016).

${ }^{45}$ S. Huberman, V. Chiloyan, R. A. Duncan, L. Zeng, R. Jia, A. A. Maznev, E. A. Fitzgerald, K. A. Nelson, and G. Chen, Phys. Rev. Mater. 1, 054601 (2017). ${ }^{46}$ C. Hua and A. J. Minnich, Phys. Rev. B 97, 014307 (2018).

${ }^{47}$ M. Forghani and N. G. Hadjiconstantinou, Appl. Phys. Lett. 114, 023106 (2019).

${ }^{48}$ G. D. Mahan and F. Claro, Phys. Rev. B 38, 1963 (1988).

${ }^{49}$ F. Claro and G. D. Mahan, J. Appl. Phys. 66, 4213 (1989).

${ }^{50}$ A. N. Grigorenko, P. I. Nikitin, D. A. Jelski, and T. F. George, Phys. Rev. B 42, 7405 (1990).

${ }^{51}$ A. N. Grigorenko, P. I. Nikitin, D. A. Jelski, and T. F. George, J. Appl. Phys. 69, 3375 (1991).

${ }^{52}$ G. D. Mahan, Phys. Rev. B 43, 3945 (1991).

${ }^{53}$ S. L. Sobolev, Phys. Rev. E 50, 3255 (1994).

${ }^{54}$ F. X. Alvarez and D. Jou, J. Appl. Phys. 103, 094321 (2008).

${ }^{55}$ Z. Banach and W. Larecki, Phys. Rev. E 78, 061137 (2008). 
${ }^{56}$ J. Ordonez-Miranda, R. Yang, and J. J. Alvarado-Gil, J. Appl. Phys. 109, 084319 (2011).

${ }^{57}$ M. Wang, X. Shan, and N. Yang, Phys. Lett. A 376, 3514 (2012).

${ }^{58}$ A. T. Ramu and Y. Ma, J. Appl. Phys 116, 093501 (2014).

${ }^{59}$ R. B. Wilson and D. G. Cahill, Nat. Commun. 5, 5075 (2014).

${ }^{60}$ Y. K. Koh, D. G. Cahill, and B. Sun, Phys. Rev. B 90, 205412 (2014).

${ }^{61}$ B. Vermeersch, J. Carrete, N. Mingo, and A. Shakouri, Phys. Rev. B 91, 085202 (2015).

${ }^{62}$ B. Vermeersch, A. M. S. Mohammed, G. Pernot, Y. R. Koh, and A. Shakouri, Phys. Rev. B 91, 085203 (2015).

${ }^{63}$ C. Hua and A. J. Minnich, J. Appl. Phys. 117, 175306 (2015).

${ }^{64}$ Y. Ezzahri, J. Ordonez-Miranda, and K. Joulain, Int. J. Heat Mass Transfer 108, 1357 (2017).

${ }^{65}$ C. Hua, X. Chen, N. K. Ravichandran, and A. J. Minnich, Phys. Rev. B 95, 205423 (2017).

${ }^{66}$ Y. Guo and M. Wang, Phys. Rev. B 97, 035421 (2018).

${ }^{67}$ Y. Guo, D. Jou, and M. Wang, Phys. Rev. B 98, 104304 (2018).

${ }^{68} \mathrm{P}$. Torres, A. Ziabari, A. Torello, J. Bafaluy, J. Camacho, X. Cartoixa, A. Shakouri, and F. X. Alvarez, Phys. Rev. Materials 2, 076001 (2018).
${ }^{69}$ P. B. Allen and V. Perebeinos, Phys. Rev. B 98, 085427 (2018).

${ }^{70}$ C. Hua, L. Lindsay, X. Chen, and A. J. Minnich, Phys. Rev. B 100, 085203 (2019).

${ }^{71}$ P. Chakraborty, B. Vermeersch, A. Shakouri, and S. Tindel, Phys. Rev. E 101, $042110(2020)$

${ }^{72}$ J. Callaway, Phys. Rev. 113, 1046 (1959).

${ }^{73}$ P. Carruthers, Rev. Mod. Phys. 33, 92 (1961).

${ }^{74}$ M. G. Holland, Phys. Rev. 132, 2461 (1963).

${ }^{75}$ R. E. Nettleton, Phys. Rev. 132, 2032 (1963).

${ }^{76}$ J. E. Parrott, Phys. Status Solidi B 48, K159 (1971).

${ }^{77}$ M. Asen-Palmer, K. Bartkowski, E. Gmelin, M. Cardona, A. P. Zhemov, A. V. Inyushkin, A. Taldenkov, V. I. Ozhogin, K. M. Itoh, and E. E. Haller, Phys. Rev. B 56, 9431 (1997).

${ }^{78}$ D. T. Morelli, J. P. Heremans, and G. A. Slack, Phys. Rev. B 66, 195304 (2002). ${ }^{79}$ Y. Ezzahri and K. Joulain, J. Appl. Phys. 112, 083515 (2012).

${ }^{80}$ R. J. Stoner and H. J. Maris, Phys. Rev. B 48, 16373 (1993).

${ }^{81}$ A. Majumdar and P. Reddy, Appl. Phys. Lett. 84, 4768 (2004).

${ }^{82}$ H. K. Lyeo and D. G. Cahill, Phys. Rev. B 73, 144301 (2006).

${ }^{83}$ R. A. Guyer and J. A. Krumhansl, Phys. Rev. 148, 766 (1966). 